\title{
DINÁMICA NUTRIMENTAL Y RENDIMIENTO DE PEPINO CULTIVADO EN HIDROPONÍA CON Y SIN RECIRCULACIÓN DE LA SOLUCIÓN NUTRITIVA
}

\section{NUTRITIONAL DYNAMICS AND YIELD OF CUCUMBER GROWN IN HYDROPONICS WITH AND WITHOUT RECIRCULATION OF THE NUTRIENT SOLUTION}

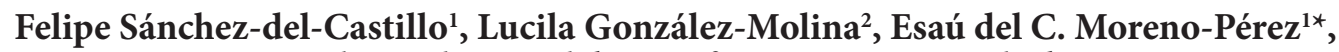 \\ Joel Pineda-Pineda ${ }^{1}$ y C. Efraín Reyes-González ${ }^{1}$
}

\begin{abstract}
${ }^{1}$ Departamento de Fitotecnia, Universidad Autónoma Chapingo. Km. 38.5 Carretera México-Texcoco. 56230, Chapingo. Estado de México, México. ${ }^{2}$ Campo Experimental Valle de México, Instituto Nacional de Investigaciones Forestales, Agrícolas y Pecuarias. Km. 13.5. Carretera Los Reyes-Texcoco. 56250, Coatlinchán, Estado de México, México.
\end{abstract}

${ }^{*}$ Autor de correspondencia (esaump10@yahoo.com.mx)

\section{RESUMEN}

Un problema frecuente en los sistemas hidropónicos en los que se recircula la solución nutritiva (sistemas cerrados) es un menor rendimiento y calidad respecto a los sistemas sin recirculación (abiertos), lo cual es atribuido a que las soluciones nutritivas se desequilibran conforme se desarrolla el cultivo, a la vez que se incrementa el riesgo de diseminación de enfermedades. El objetivo de la investigación fue evaluar la factibilidad de sistemas hidropónicos cerrados para alcanzar rendimientos de pepino (Cucumis sativus $\mathrm{L}$.) similares a los que se obtienen con sistemas abiertos, mediante el acortamiento del ciclo de cultivo. Se compararon tres modalidades de sistemas cerrados (raíz flotante, cultivo en bolsa con sustrato y cultivo en cama con sustrato) y dos abiertos (cultivo en bolsa y en cama con sustrato), bajo un diseño de bloques completos al azar con cinco repeticiones. Se encontró que tanto el crecimiento de la planta como el rendimiento de fruto de pepino fueron similares entre los sistemas con y sin recirculación de la solución nutritiva en bolsa y cama, pero con raíz flotante se alcanzó mayor acumulación de materia seca y mayor rendimiento por unidad de superficie. Los sistemas cerrados permitieron un ahorro superior a $20 \%$ en agua y de $25 \%$ en nutrimentos, en comparación con los sistemas abiertos.

Palabras clave: Cucumis sativus, raíz flotante, despunte, solución nutritiva.

\section{SUMMARY}

A common problem in hydroponics systems in which the nutrient solution is recirculated (closed systems) is a lower yield and quality over systems without recirculation (open system), which is attributed to imbalances of nutrient solutions and to an increase in the risk of spread of root diseases as the crop is developing. This research evaluated the possibility of closed hydroponic systems to achieve cucumber (Cucumis sativus L.) yields similar to those obtained with open systems, by shortening the growing cycle. Three types of closed systems (floating hydroponics, cultivation in bed with substrate and cultivation in bag with substrate) and two open systems (culture in bag and in bed with substrate), were compared under a complete randomized block design with five replications. It was found that both plant growth and fruit yield of cucumber were similar between closed and open systems in bag and bed, but with floating hydroponics a higher dry matter accumulation and yield per unit area were reached. Closed systems allowed savings of over $20 \%$ in water and $25 \%$ in nutrients compared to open systems.

Index words: Cucumis sativus, floating hydroponics, trimming, nutrient solution.

\section{INTRODUCCIÓN}

La agricultura protegida por los beneficios que ofrece (altos rendimientos y calidad, mayores niveles de sanidad e inocuidad de los productos obtenidos, seguridad en la producción con cierta independencia del clima, acceso a mejores mercados y potencial de alta rentabilidad económica), está creciendo en México. Actualmente están bajo cubierta 20,000 ha, de las cuales 12,000 son de invernaderos y 8000 de estructuras denominadas casa-sombra (AMHPAC, 2013; Ponce, 2013). En estas condiciones la hidroponía le gana más terreno a la producción en suelo debido a que se logra mayor eficiencia y control del riego y la nutrición mineral, ausencia inicial de plagas, enfermedades y malezas, facilidad de esterilización de los sustratos, posibilidad de usar aguas duras o con mayor salinidad, mayor rendimiento y calidad, y más sanidad e inocuidad, entre otras (Cánovas y Magán, 2003; Alarcón, 2006; Raviv y Lieth, 2008).

En la mayoría de los sistemas hidropónicos establecidos se aplica el riego por goteo con una solución nutritiva que contiene fertilizantes disueltos con todos los nutrientes minerales esenciales para las plantas, en concentraciones óptimas para su crecimiento y desarrollo. Para que las plantas de pepino (Cucumis sativus L.) crezcan sin limitaciones nutricionales, la solución nutritiva debe tener un $\mathrm{pH}$ entre 5.5 a 6.5, una conductividad eléctrica (CE) entre 1.5 y $3 \mathrm{dS} \mathrm{m}^{-1}$, y los nutrimentos minerales deben estar disociados en proporciones y concentraciones que eviten precipitados y antagonismos (Adams, 2004). La planta modifica el consumo de nutrimentos en función de sus fases de crecimiento y desarrollo, condiciones climáticas, y características de la solución nutritiva como la $\mathrm{CE}, \mathrm{pH}$ y 
oxígeno disuelto (Terabayashi et al., 2004; Jones, 2005; Sonneveld y Voogt, 2009).

Cuando la solución drenada no se reutiliza y se permite la infiltración en el sitio o se conduce fuera del invernadero, al sistema hidropónico se le conoce como abierto; por el contrario, si se recoge para volverse a usar en el cultivo, previa esterilización y ajuste de $\mathrm{pH}, \mathrm{CE}$ y concentración de nutrimentos, se le llama sistema cerrado (Alarcón, 2006).

Debido al encarecimiento de los fertilizantes (Huang, 2009) y al impacto negativo en el ambiente (Giuffrida y Leonardi, 2009; Nakano et al., 2010; Massa et al., 2010), en hidroponía se buscan sistemas más eficientes. Por ello los sistemas hidropónicos abiertos empiezan a ser sustituidos por los cerrados (Alarcón, 2006). Éstos últimos presentan ventajas importantes respecto a los primeros: ahorro de agua y fertilizantes, y menor impacto ambiental al evitar que grandes cantidades de minerales contaminen ríos, lagos, mantos freáticos y mares (Giuffrida y Leonardi, 2009; Pardossi et al., 2009; Nakano et al., 2010; Massa et al., 2010).

Al sistema cerrado también se le han detectado desventajas, como: incremento gradual de la CE de la solución nutritiva con el paso del tiempo, desbalance de la solución nutritiva y mayor riesgo de dispersar enfermedades que atacan a la raíz (Tüzel et al., 2009; Van-Os, 2009; Massa et al., 2010). El desbalance de la solución nutritiva se genera por la acumulación de los iones menos consumidos por la planta $\left(\mathrm{SO}_{4}^{2-}, \mathrm{Ca}^{2+}\right.$ y $\left.\mathrm{Mg}^{2+}\right)$, lo que rompe el equilibrio de nutrimentos y en la mayoría de las veces incrementa la CE a niveles que afectan el crecimiento y rendimiento (Savvas et al., 2009), lo que con frecuencia ha obligado a desechar la solución nutritiva.

En la práctica comercial con sistemas hidropónicos cerrados, entre más largo es el ciclo de cultivo, mayor es la posibilidad de que aparezcan enfermedades en la raíz y desequilibrios en la solución nutritiva, lo que eventualmente puede afectar el rendimiento respecto a sistemas sin recirculación. Por ello con frecuencia se reportan rendimientos menores en sistemas cerrados respectos a los abiertos en cultivos de ciclo largo como tomate (Solanum lycopersicum L.), pimiento (Capsicum annuum L.) o pepino en los que durante varios meses coexisten etapas de crecimiento vegetativo con reproductivo (Savvas et al., 2009; Nakano et al., 2010).

En la Universidad Autónoma Chapingo se ha estado trabajando en el desarrollo de un sistema de producción de pepino para acortar su ciclo de trasplante a fin de cosechar en un máximo de dos meses. El sistema se basa en hacer el trasplante con plántulas de 30 d de edad en vez de 20 d en los sistemas de producción que usan charolas en semilleros, efectuar un despunte (eliminación de la yema terminal) de las plantas a $1 \mathrm{~m}$ de altura, y plantar a una alta densidad de población para compensar el menor rendimiento que se obtiene por planta, lo cual es posible por la menor área foliar que desarrolla cada planta con el despunte (Sánchezdel-Castillo et al., 2006; Ortiz et al., 2009).

El objetivo del presente trabajo fue estudiar, para sistemas con recirculación de la solución nutritiva, si al acortar el ciclo de cultivo de pepino a dos meses desde trasplante a fin de cosecha, y plantando en alta densidad de población, se puede escapar a desequilibrios importantes de la solución nutritiva como para obtener al menos el mismo rendimiento y calidad que en los sistemas sin recirculación. Con ello se pretende contribuir a generar un sistema de producción de pepino que aproveche los beneficios de recircular la solución nutritiva sin arriesgar el rendimiento y calidad por los desequilibrios nutricionales, y sin recurrir a costosos y frecuentes análisis químicos para corregirlos.

\section{MATERIALES Y MÉTODOS}

El experimento se estableció en un invernadero con cubierta de polietileno localizado en el municipio de Texcoco, Estado de México, a $19^{\circ} 29^{\prime} \mathrm{N}, 90^{\circ} 53^{\prime} \mathrm{O}$ y una altitud de $2251 \mathrm{~m}$.

Se usó el híbrido de pepino americano variedad 'Alcázar'. Para el trasplante se utilizaron plántulas de $30 \mathrm{~d}$ de edad, provenientes de charolas de 200 cavidades, en las que el sustrato fue una mezcla de turba ("peat moss") y perlita a partes iguales. Las plántulas fueron regadas con agua hasta la emergencia, y en los siguientes días y hasta el trasplante con una solución nutritiva que contenía 50 \% de la solución nutritiva propuesta por Sánchez-del-Castillo y Escalante (1988) y que se indica más adelante.

Se compararon tres modalidades de sistemas cerrados (raíz flotante, cultivo en bolsa con sustrato y cultivo en cama con sustrato) y dos abiertos (cultivo en bolsa y en cama con sustrato), los cuales se describen a continuación:

Sistema de raíz flotante (raíz flotante). Se construyeron camas de madera ( $1.9 \mathrm{~m}$ de largo por $0.9 \mathrm{~m}$ de ancho y $0.3 \mathrm{~m}$ de profundidad), cuyo interior se cubrió con plástico negro calibre 1000. Las camas se llenaron con $400 \mathrm{~L}$ de solución nutritiva y se cubrieron con una placa de unicel (poliestireno expandido) que flotaba sobre ella. En el momento del trasplante las plántulas, con su cepellón, se pusieron dentro de vasos de plástico de $50 \mathrm{~mL}$ a los cuales se le retiró la base inferior con el propósito de sostener la plántula y que sólo las raíces quedaran sumergidas. La solución nutritiva se oxigenó continuamente con dos bombas de aire marca Resum ${ }^{\circledR}, A C-9602$ (México). En este tratamiento el agua 
que las plantas transpiraban se restablecía todos los días al final del día, y diariamente se medía el pH y en su caso se ajustaba a un valor entre 5.5 y 6.5. También diariamente se registraba el valor de la $\mathrm{CE}$.

Sistema de bolsas con arena de tezontle (partículas de 1 a $3 \mathrm{~mm}$ ) y recirculación de la solución nutritiva drenada (bolsa con recirculación). Las bolsas tenían una capacidad de $15 \mathrm{~L}$, de color negro por dentro y blanco por fuera, se instalaron sobre canales de PVC (policloruro de vinilo) a fin de recolectar la solución drenada. Los canales se pusieron con una ligera pendiente para que el drenaje de las bolsas se dirigiera hacia una cubeta de 19 L donde cada día se medía su volumen, $\mathrm{pH}$ y $\mathrm{CE}$. La solución nutritiva que se colectaba se conducía a un tinaco de $400 \mathrm{~L}$.

Sistema de bolsas con arena de tezontle sin recirculación de la solución nutritiva drenada (bolsa sin recirculación). El sistema era similar al tratamiento anterior, excepto que la solución drenada, después de medirle su volumen, pH y CE, se desechaba.

Camas con arena de tezontle y recirculación de la solución nutritiva drenada (cama con recirculación). Se construyeron camas con polietileno negro calibre 1000 en el fondo y con tablas de madera en los costados, con dimensiones de $1.9 \mathrm{~m}$ de largo por $0.9 \mathrm{~m}$ de ancho y $0.3 \mathrm{~m}$ de altura. El plástico negro sobresalió de las camas $30 \mathrm{~cm}$ hacia el pasillo para formar un canal para recuperar la solución del drenaje. Para rellenar la cama se depositó una capa de 5 $\mathrm{cm}$ de grava de tezontle (partículas de 4 a $8 \mathrm{~cm}$ ) en el fondo y así facilitar el drenaje, y encima otra capa de $25 \mathrm{~cm}$ de arena de tezontle rojo (partículas de $1 \mathrm{a} 3 \mathrm{~mm}$ ) como sustrato. Se acolchó la superficie con un polietileno bicolor, blanco la parte superior y negro la parte inferior. Para recuperar el drenaje se perforó el plástico en la parte baja de la cama y se condujo por gravedad a cubetas de $19 \mathrm{~L}$. A la solución drenada se le midió diariamente su volumen, $\mathrm{pH}$ y CE. La solución nutritiva que se colectaba se conducía a un tinaco de $400 \mathrm{~L}$.

Camas con arena de tezontle sin recirculación de la solución nutritiva drenada (cama sin recirculación). El sistema era similar al tratamiento anterior, excepto que se desechaba la solución drenada, después de medirle su volumen, $\mathrm{pH}$ y CE.

Se usó un diseño experimental de bloques completos al azar con cinco tratamientos y cinco repeticiones. La unidad experimental fue de $1.7 \mathrm{~m}^{2}$ útiles $(1.9 \times 0.9 \mathrm{~m})$, donde se establecieron 18 plantas distribuidas en tres hileras, separadas a una distancia de $30 \mathrm{~cm}$ entre plantas y entre hileras, con lo que se tuvo una densidad de 6 plantas por $\mathrm{m}^{2}$ de invernadero.
El volumen aplicado de riego con solución nutritiva dependió de las condiciones climáticas y etapa fenológica del cultivo, pero se procuraba aplicar un sobre riego de 20 a $30 \%$ del volumen calculado en cada riego. La solución nutritiva que se usó en todos los tratamientos presentaba las siguientes concentraciones de nutrimentos $\left(\mathrm{en} \mathrm{mg} \mathrm{L}^{-1}\right)$ : $\mathrm{N}=$ $140, \mathrm{P}=40, \mathrm{~K}=175, \mathrm{Ca}=140, \mathrm{Mg}=40, \mathrm{~S}=140, \mathrm{Fe}=1.5$, $\mathrm{Mn}=0.5, \mathrm{~B}=0.5, \mathrm{Cu}=0.1, \mathrm{y} \mathrm{Zn}=0.1$ que corresponde a la recomendada por Sánchez-del-Castillo y Escalante (1988). Como fuentes se usaron los siguientes fertilizantes comerciales: nitrato de calcio, sulfato de potasio, ácido fosfórico a $85 \%$, sulfato de magnesio, quelato de fierro, sulfato de manganeso, tetraborato de sodio, sulfato de cobre y sulfato de zinc. El análisis del agua de riego indicó una $\mathrm{CE}$ de 0.2 $\mathrm{dS} \mathrm{m}^{-1}$ y concentración de cloro y sodio de 10 y $16 \mathrm{mg} \cdot \mathrm{L}^{-1}$, respectivamente, por lo que se considera de buena calidad.

Se utilizó un sistema de tutorado para sostener a las plantas. En todos los sistemas las plantas fueron despuntadas para dejarlas a $1 \mathrm{~m}$ de altura. La Figura 1 ilustra el aspecto de las plantas de pepino en este estudio.

Los tratamientos de recirculación fueron abastecidos de solución por su propio tinaco de $1000 \mathrm{~L}$ mientras que los tratamientos de cama y bolsa sin recirculación compartían un solo tinaco de $1000 \mathrm{~L}$. En cada tratamiento, excepto el de raíz flotante (RF), se usó una bomba de $1 / 2 \mathrm{HP}$, un temporizador STEREN $®$ modelo TEMP-085, un filtro de anillos (marca Irritec ${ }^{\circledR}$ de 120 "mesh" o $130 \mu \mathrm{m}$ ), un medidor de flujo, tubería de $2.54 \mathrm{~cm}$ y cinta de goteo con gasto por emisor de $1 \mathrm{~L} \mathrm{~h}^{-1}$. A cada planta le correspondió un gotero.

En los sistemas con y sin recirculación todos los días se midió el volumen de solución nutritiva aportada con el medidor de flujo, y la cantidad de solución nutritiva colectada en las cubetas con una probeta de $1000 \mathrm{~mL}$; también se determinó el pH y CE con un medidor portátil Hanna ${ }^{\circledR}$, modelo HI 98130. En el sistema de raíz flotante se registró el agua aportada así como su pH y CE. El drenaje de los sistemas sin recirculación se vertió fuera del invernadero mientras que en los de recirculación se colectó en tinacos de $400 \mathrm{~L}$. A los 17, 25, 35, 43 y $58 \mathrm{~d}$ después del trasplante (ddt), cuando se acumulaban cerca de 400 L de solución drenada, se tomó una muestra de cada tinaco junto con una muestra de cada repetición del sistema de RF.

Para analizar la concentración de nutrientes $\left(\mathrm{N}^{-} \mathrm{NO}_{3}\right.$ $\mathrm{y} \mathrm{K}^{+}$) se utilizó un electrodo de ion selectivo (Thermo Scientific ${ }^{\circledR}$, modelo Orion 4 Star) y para $\mathrm{P}$ se usó la técnica colorimétrica con el método de molibdobanadato (Chapman y Pratt, 1973). Conocida la composición de la solución nutritiva, ésta se filtraba y desinfectaba con una lámpara UV (Philips® de 25 watts y un flujo de $22.8 \mathrm{~L}$ $\mathrm{min}^{-1}$ ) antes de pasarla al tinaco de su tratamiento (bolsa o 

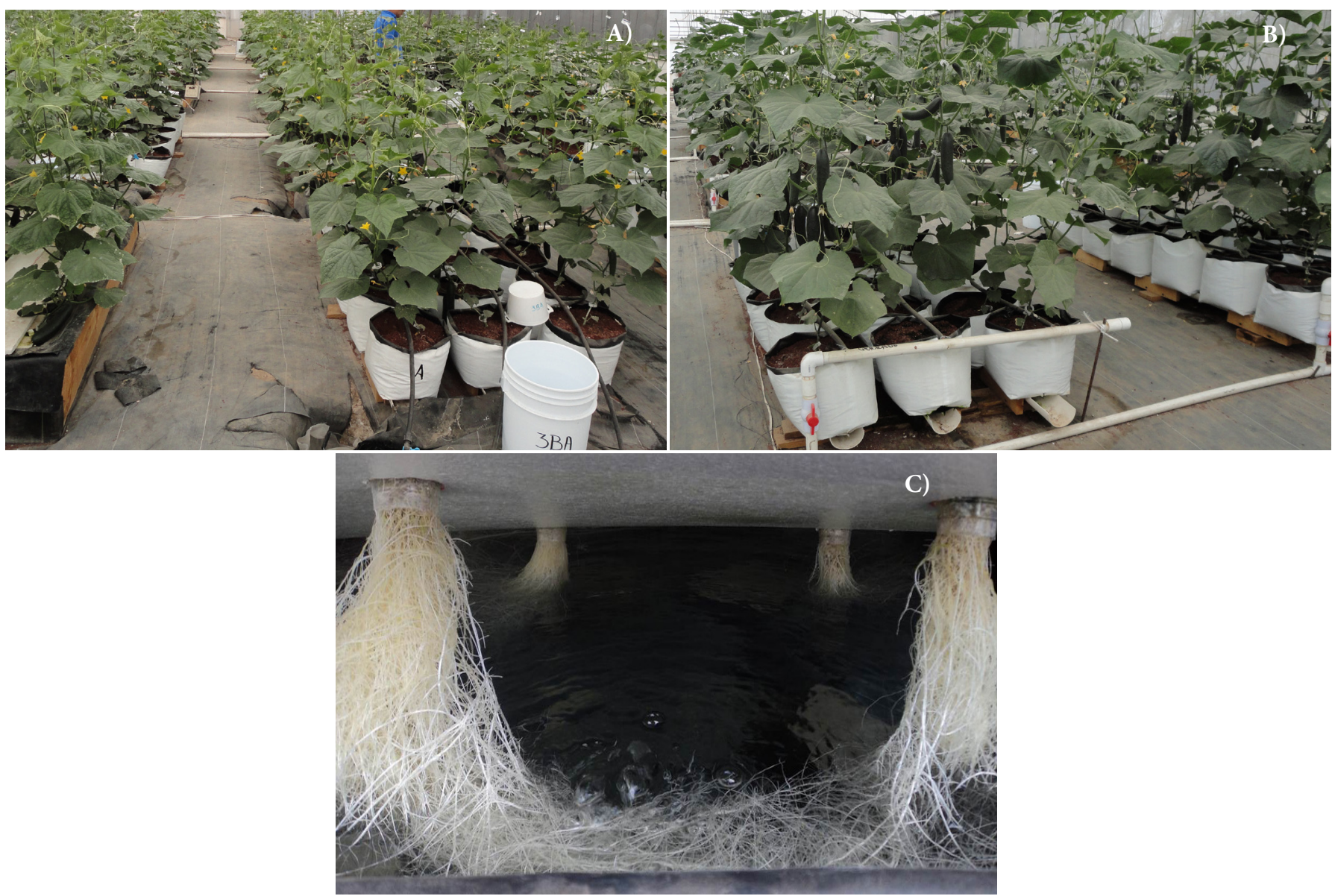

Figura 1. Plantas de pepino crecidas en invernadero con diversos sistemas hidropónicos. A) Plantas en etapa vegetativa; B) Plantas en etapa reproductiva; C) Raíces en sistema flotante.

cama con recirculación). En el tinaco la solución nutritiva se ajustaba a las concentraciones iniciales de N, P, K y de CE. En las tinas de RF la solución nutritiva se ajustó de manera individual en cada repetición.

A la solución nutritiva que drenaba de los tratamientos de bolsa y cama con recirculación, se le medía su CE y se le añadía agua hasta equilibrarla con la de la solución nutritiva original ( 2 a $2.5 \mathrm{dS} \mathrm{m}^{-1}$ ); en ese momento se medía la concentración de $\mathrm{N}, \mathrm{P}$ y K, y cuando fue necesario se agregó fertilizante para reponer el o los elementos faltantes; finalmente se aforaba el tinaco con solución nutritiva normal.

Las variables medidas fueron: a) diámetro de tallo, a los 23, 36 y $46 \mathrm{ddt}$; b) área foliar por planta, medida con un integrador de área foliar (LICOR-300® Lincoln, NE, USA) a los 23, 36 y $46 \mathrm{ddt}$; c) peso seco total por planta, obtenido después de secar en estufa a $70^{\circ} \mathrm{C}$ hasta peso constante, a los 23, 36, 46 y $55 \mathrm{ddt}$, medida en una planta por unidad experimental de cada tratamiento y cada repetición; d) rendimiento por unidad de superficie; y e) número de frutos cosechados por unidad de superficie. El último corte se rea- lizó a los 58 ddt.

Para estimar el ahorro de agua y fertilizantes de los sistemas cerrados con respecto a los abiertos se hicieron las siguientes mediciones: a) volumen de agua gastado en el ciclo de cultivo; b) consumo de agua por planta en el ciclo de cultivo; c) ahorro de agua de los sistemas cerrados de bolsa y cama con respecto a sus similares en sistema abierto; d) eficiencia en el uso del agua (litros de agua consumidos por kilogramo de fruto producido); e) consumo aparente de $\mathrm{N}$, P y K por las plantas a lo largo del ciclo; f) cantidad de N, P y K desechada a lo largo del ciclo; g) cantidad de N, P y K retenida en el sustrato al final del ciclo; h) ahorro de N, P y $\mathrm{K}$ con respecto a los sistemas abiertos; e i) cambios del $\mathrm{pH}$ y de la CE a lo largo del ciclo.

\section{RESULTADOS Y DISCUSIÓN}

\section{Variables indicadoras del crecimiento}

En las distintas fechas de muestreo, el diámetro de tallo tendió a ser mayor en el sistema de raíz flotante (RF) que en 
Cuadro 1. Comparación de medias para variables indicadoras del crecimiento medidas durante el ciclo de cultivo de pepino crecido en diferentes sistemas hidropónicos.

\begin{tabular}{|c|c|c|c|c|}
\hline Muestreo (ddt) & Sistema hidropónico & $\begin{array}{l}\text { Diámetro de } \\
\text { tallo }(\mathrm{mm})\end{array}$ & $\begin{array}{c}\text { Área foliar por } \\
\text { planta }\left(\mathrm{dm}^{2}\right)\end{array}$ & $\begin{array}{l}\text { Peso seco por } \\
\text { planta }(\mathrm{g})\end{array}$ \\
\hline \multirow[t]{6}{*}{23} & Raíz flotante & $4.36 \mathrm{a}$ & $14.3 \mathrm{ab}$ & $10.4 \mathrm{a}$ \\
\hline & Bolsa sin recirculación & $3.98 \mathrm{ab}$ & $19.2 \mathrm{a}$ & $11.6 \mathrm{a}$ \\
\hline & Bolsa con recirculación & $3.93 \mathrm{ab}$ & $16.2 \mathrm{ab}$ & $9.4 \mathrm{a}$ \\
\hline & Cama sin recirculación & $3.85 \mathrm{~b}$ & $17.9 \mathrm{a}$ & $12.4 \mathrm{a}$ \\
\hline & Cama con recirculación & $3.84 \mathrm{~b}$ & $11.7 \mathrm{~b}$ & $9.3 \mathrm{a}$ \\
\hline & DMS & 0.46 & 6.0 & 6.04 \\
\hline \multirow[t]{6}{*}{36} & Raíz flotante & $4.85 \mathrm{a}$ & 39.9 a & $66.2 \mathrm{a}$ \\
\hline & Bolsa sin recirculación & $4.67 \mathrm{a}$ & $33.8 \mathrm{~b}$ & $55.3 \mathrm{ab}$ \\
\hline & Bolsa con recirculación & $4.50 \mathrm{a}$ & $31.9 \mathrm{~b}$ & $49.3 \mathrm{ab}$ \\
\hline & Cama sin recirculación & $4.34 \mathrm{a}$ & $36.0 \mathrm{ab}$ & $46.7 \mathrm{~b}$ \\
\hline & Cama con recirculación & $4.33 \mathrm{a}$ & $39.7 \mathrm{a}$ & $49.4 \mathrm{~b}$ \\
\hline & DMS & 0.61 & 6.0 & 19.2 \\
\hline \multirow[t]{6}{*}{46} & Raíz flotante & $8.12 \mathrm{a}$ & $39.5 \mathrm{~b}$ & $84.1 \mathrm{a}$ \\
\hline & Bolsa sin recirculación & $7.01 \mathrm{~b}$ & $49.4 \mathrm{a}$ & $74.9 \mathrm{ab}$ \\
\hline & Bolsa con recirculación & $6.87 \mathrm{~b}$ & $43.8 \mathrm{ab}$ & $59.0 \mathrm{bc}$ \\
\hline & Cama sin recirculación & $6.75 \mathrm{~b}$ & $43.4 \mathrm{ab}$ & $60.0 \mathrm{bc}$ \\
\hline & Cama con recirculación & $6.89 \mathrm{~b}$ & $39.3 \mathrm{~b}$ & $52.6 \mathrm{c}$ \\
\hline & DMS & 0.45 & 7.1 & 21.5 \\
\hline \multirow[t]{6}{*}{55} & Raíz flotante & - & - & $89.7 \mathrm{a}$ \\
\hline & Bolsa sin recirculación & - & - & $79.6 \mathrm{ab}$ \\
\hline & Bolsa con recirculación & - & - & $68.5 \mathrm{~b}$ \\
\hline & Cama sin recirculación & - & - & $63.2 \mathrm{~b}$ \\
\hline & Cama con recirculación & - & - & $66.3 \mathrm{~b}$ \\
\hline & DMS & - & - & 20.1 \\
\hline
\end{tabular}

DMS = diferencia mínima significativa. Medias con la misma letra en una columna no presentan diferencia significativa (Tukey, 0.05). $\mathrm{ddt}=$ días después del trasplante.

Cuadro 2. Comparación de medias para variables de rendimiento y sus componentes primarios en pepino crecido bajo diferentes sistemas hidropónicos.

\begin{tabular}{lccc}
\hline Sistema hidropónico & Peso medio de fruto $(\mathrm{g})$ & Frutos m ${ }^{-2}$ & $\begin{array}{c}\text { Rendimiento } \\
\left(\mathrm{kg} \mathrm{m}^{-2}\right)\end{array}$ \\
\hline Raíz flotante & $270 \mathrm{a}$ & $44 \mathrm{a}$ & $11.80 \mathrm{a}$ \\
Bolsa sin recirculación & $268 \mathrm{a}$ & $35 \mathrm{ab}$ & $9.29 \mathrm{~b}$ \\
Bolsa con recirculación & $273 \mathrm{a}$ & $34 \mathrm{~b}$ & $9.16 \mathrm{~b}$ \\
Cama sin recirculación & $274 \mathrm{a}$ & $38 \mathrm{ab}$ & $10.23 \mathrm{ab}$ \\
Cama con recirculación & $275 \mathrm{a}$ & $43 \mathrm{a}$ & $11.58 \mathrm{a}$ \\
DMS & 33.89 & 10.18 & 1.79 \\
\hline
\end{tabular}

DMS = diferencia mínima significativa. Medias con la misma letra en una columna no presentan diferencia significativa (Tukey, 0.05). 
los demás tratamientos, diferencia que fue estadísticamente superior a los $46 \mathrm{ddt}$ (Cuadro 1). Entre los sistemas con y sin recirculación en bolsas y entre sistemas de camas abiertos y cerrados, no hubo diferencias en altura de planta ni en área foliar por planta.

En cambio, a partir de los 36 ddt el peso seco por planta fue más alto en el sistema de RF y estadísticamente superior en los tres últimos muestreos, que el registrado en los sistemas de cama con y sin recirculación. El único sistema que mantuvo igualdad estadística con la RF fue el de bolsa sin recirculación.

La acumulación de biomasa observada en las plantas crecidas en el tratamiento RF se atribuye a que en este sistema no hay limitaciones de agua para la raíz y se mantiene más estable la concentración de nutrientes en la rizosfera, ya que los cambios en concentración ocurren lentamente por la gran cantidad de solución nutritiva presente por planta. En cambio, en los otros sistemas la cantidad de agua y las concentraciones de nutrientes en la rizosfera varían considerablemente entre un riego y otro debido a la limitada capacidad de retención de solución nutritiva por el contenedor, lo que afecta más la absorción (Silber y Bar-tal, 2008).

$\mathrm{Al}$ inicio del experimento la CE fue de $2.25 \mathrm{dS} \mathrm{m}^{-1}$. En el sistema de RF, la CE disminuyó paulatinamente hasta alcanzar un mínimo de $1.85 \mathrm{dS} \mathrm{m}^{-1}$ debido a que el agua que se perdía por la transpiración era sustituida regularmente con agua pura, y sólo cada 10 a 15 d se reponían nutrientes.

En los tratamientos con sustrato, con y sin recirculación, los valores de CE en el drenaje se incrementaron con el tiempo hasta alcanzar valores de $2.6 \mathrm{dS} \mathrm{m}^{-1}$ al final del ciclo. Esto se explica como consecuencia de una acumulación progresiva de sales en el sustrato debido a la evapotranspiración, de manera que al aplicar la solución nutritiva el drenaje excedente arrastra parte de las sales presentes en el sustrato (Castellanos y Borbón, 2009).

Se esperaba que el incremento de la CE observado en los tratamientos de bolsa y de cama con recirculación afectaran el crecimiento (Savvas et al., 2009), pero no fue así ya que el pepino se condujo en un ciclo corto de 2 meses, lo que contribuyó a que la CE de la solución nutritiva no aumentara en magnitud importante como para afectar el crecimiento.

\section{Rendimiento y sus componentes}

El rendimiento por unidad de superficie fue estadísticamente mayor en los tratamientos de RF y de camas con recirculación, que en los sistemas de cultivo en bolsa, tanto abiertos como cerrados (Cuadro 2). Entre los tratamientos de cama con y sin recirculación no hubo diferencias signi- ficativas, ni tampoco entre los de bolsa con y sin recirculación.

Las diferencias en rendimiento se atribuyen al número de frutos cosechados por unidad de superficie, ya que el peso medio de frutos fue similar en todos los tratamientos (Cuadro 2), lo cual coincide con Ortiz et al. (2009) quienes señalaron que en pepino sometido a despuntes tempranos y a alta densidad de población en un ambiente poco restrictivo, el número de frutos por planta fue el principal componente que afectó el rendimiento. Por su parte, Pardossi et al. (2009) reportaron que un elevado nivel de salinidad en el sistema con recirculación reduce el rendimiento.

Sin embargo, en el presente estudio no se encontró diferencia entre sistemas con y sin recirculación de la solución nutritiva, debido a que en los sistemas de producción con recirculación aquí evaluados en el ciclo de cultivo del pepino fue de 2 meses por el despunte temprano (contra más de 4 meses en los sistemas convencionales), tiempo insuficiente para que se desajustara de manera importante la solución nutritiva, tanto en su CE como en la proporción relativa de nutrimentos.

El mayor rendimiento y número de frutos por unidad de superficie en RF podría explicarse por un ambiente más estable en la rizosfera, sobre todo en comparación con el sistema de bolsa que tiene menos sustrato por planta, porque en este último ocurren más fluctuaciones en temperatura, $\mathrm{CE}$, contenido de humedad y concentración de nutrimentos en la raíz, que pueden provocar estrés en las plantas (Liang et al., 2006).

Cabe destacar que por lo corto del ciclo de las plantas podadas y el ambiente controlado del invernadero, es posible obtener hasta seis ciclos de cultivo por año lo que permitiría alta productividad anual, como previamente señalaron Ortiz et al. (2009).

\section{Uso y eficiencia del agua y nutrientes}

El reciclar la solución nutritiva significó ahorros importantes de agua y nutrimentos sin afectar el rendimiento (Cuadro 3). En los tratamientos de bolsas con recirculación el ahorro fue de $23 \%$ con respecto a los de bolsas sin recirculación, mientras que para cama con recirculación el ahorro fue de $20 \%$ con respecto a la cama sin recirculación. Con recirculación también hubo un ahorro superior a $30 \%$ en nutrimentos ( $\mathrm{K}, \mathrm{N}$ y $\mathrm{P})$, comparado con los sistemas sin recirculación (Cuadro 4).

Con el uso de sistemas de recirculación se han reportado ahorros importantes en fertilizantes, pero con una reducción en el rendimiento de fruto atribuida a desequilibrios 
Cuadro 3. Indicadores de eficiencia en el uso del agua en plantas de pepino a los 55 días después del trasplante cultivadas en diferentes sistemas hidropónicos.

\begin{tabular}{lccccc}
\hline Sistema hidropónico & $\begin{array}{c}\text { Volumen de agua } \\
\text { gastado }\left(\mathrm{L} \mathrm{m}^{-2}\right)\end{array}$ & $\begin{array}{c}\text { Ahorro de } \\
\text { agua }(\%)\end{array}$ & $\begin{array}{c}\text { Desechado } \\
\left(\mathrm{L} \mathrm{m}^{-2}\right)\end{array}$ & $\begin{array}{c}\text { Consumo de agua } \\
(\mathrm{L} / \mathrm{planta})\end{array}$ & $\begin{array}{c}\text { Eficiencia de uso } \\
\text { del agua }\left(\mathrm{L} \mathrm{kg}^{-1}\right)\end{array}$ \\
\hline Raíz flotante & 462 & - & - & 70 & 39.2 \\
Bolsa sin recirculación & 486 & - & 182 & 81 & 52.3 \\
Bolsa con recirculación & 376 & 22.6 & - & 81 & 41.0 \\
Cama sin recirculación & 484 & - & - & 81 & 47.3 \\
Cama con recirculación & 387 & 20.2 & 182 & 83 & 33.4 \\
\hline
\end{tabular}

Volumen de agua gastado = volumen de agua aplicado - volumen de agua desechado. Ahorro de agua con respecto al sistema abierto correspondiente. Eficiencia de uso del agua $=$ volumen de agua gastado/rendimiento.

Cuadro 4. Uso de potasio, nitrógeno y fósforo en plantas de pepino cultivadas en diferentes sistemas hidropónicos.

\begin{tabular}{|c|c|c|c|c|c|c|c|c|}
\hline \multirow{2}{*}{ Nutrimento } & \multirow{2}{*}{$\begin{array}{c}\text { Sistema } \\
\text { hidropónico }\end{array}$} & \multirow{2}{*}{$\begin{array}{c}\text { Nutrimento } \\
\text { aplicado } \\
\left(\mathrm{g} \mathrm{m}^{-2}\right)\end{array}$} & \multicolumn{2}{|c|}{ Drenaje desechado } & \multicolumn{2}{|c|}{$\begin{array}{l}\text { Solución } \\
\text { consumida }\end{array}$} & \multicolumn{2}{|c|}{$\begin{array}{l}\text { Solución retenida } \\
\text { en el sustratos }\end{array}$} \\
\hline & & & $\left(\mathrm{g} \mathrm{m}^{-2}\right)$ & $(\%)$ & $\left(\mathrm{g} \mathrm{m}^{-2}\right)$ & $(\%)$ & $\left(\mathrm{g} \mathrm{m}^{-2}\right)$ & $(\%)$ \\
\hline \multirow[t]{5}{*}{$\mathrm{K}$} & Raíz flotante & 108.5 & - & - & 26.1 & 24.1 & - & - \\
\hline & Bolsa sin recirculación & 140.0 & 52.4 & 37.4 & 21.2 & 15.1 & 66.4 & 47.4 \\
\hline & Bolsa con recirculación & 112.0 & 7.8 & 7.0 & 19.1 & 17.1 & 85.1 & 75.9 \\
\hline & Cama sin recirculación & 137.2 & 51.4 & 37.5 & 21.9 & 16.0 & 63.9 & 46.5 \\
\hline & Cama con recirculación & 105.0 & 7.6 & 7.2 & 18.7 & 17.8 & 78.7 & 74.9 \\
\hline \multirow[t]{5}{*}{$\mathrm{N}$} & Raíz flotante & 87.6 & - & - & 18.0 & 20.5 & - & - \\
\hline & Bolsa sin recirculación & 109.8 & 41.1 & 37.4 & 14.5 & 13.2 & 54.1 & 49.3 \\
\hline & Bolsa con recirculación & 83.2 & 8.0 & 9.6 & 12.5 & 15.0 & 62.6 & 75.2 \\
\hline & Cama sin recirculación & 106.7 & 40.0 & 37.5 & 16.7 & 15.6 & 49.9 & 46.8 \\
\hline & Cama con recirculación & 85.0 & 9.2 & 10.0 & 17.1 & 20.1 & 58.6 & 68.9 \\
\hline \multirow[t]{5}{*}{$\mathrm{P}$} & Raíz flotante & 18.1 & - & - & 6.2 & 34.2 & - & - \\
\hline & Bolsa sin recirculación & 22.4 & 8.3 & 37.4 & 5.6 & 25.0 & 8.3 & 51.8 \\
\hline & Bolsa con recirculación & 17.5 & 1.1 & 6.2 & 4.4 & 25.1 & 5.7 & 51.1 \\
\hline & Cama sin recirculación & 23.4 & 8.7 & 37.5 & 5.7 & 24.3 & 8.2 & 52.5 \\
\hline & Cama con recirculación & 18.7 & 1.3 & 6.8 & 5.2 & 27.8 & 6.4 & 54.7 \\
\hline
\end{tabular}

'En el sistema de raíz flotante, el porcentaje que es considerado como retenido hace referencia a las precipitaciones químicas.

químicos o a contaminación biológica de la solución nutritiva recirculante (Giuffrida et al., 2003; Parra et al., 2009), aunque también hay reportes en los que el rendimiento no disminuyó debido a un control más efectivo (Giuffrida y Leonardi, 2009; Nakano et al., 2010).

Como consecuencia de la reutilización de la solución nutritiva, los sistemas cerrados fueron más eficientes en el uso de agua, con valores de 33.4, 39.2, $41.0 \mathrm{~L}$ de agua gastada por cada kg de fruto producido en cama cerrada, raíz flotante y bolsa cerrada, respectivamente, contra 47.3 y $52.3 \mathrm{~L}$ $\mathrm{kg}^{-1}$ requeridos en los tratamientos de cama y bolsa abierta, respectivamente (Cuadro 4), lo cual es consistente con lo reportado por Parra et al. (2009).
Como era de esperarse, en el sistema hidropónico se observó una relación directa entre la absorción nutrimental (Cuadro 4) y el rendimiento del cultivo. Las plantas de los tratamientos de raíz flotante y de cama con recirculación fueron las que más rindieron (11.80 y $11.58 \mathrm{~kg} \mathrm{~m}^{-2}$, respectivamente), y también tuvieron la mayor absorción nutrimental; en RF se consumieron 26.1, 18 y $6.2 \mathrm{~g} \mathrm{~m}^{-2}$ de K, N y $\mathrm{P}$, respectivamente, y para el de cama con recirculación el consumo fue de 18.7, 17.1, $5.2 \mathrm{~g} \mathrm{~m}^{-2}$. En contraste, las plantas de los tratamientos con bolsa, con y sin recirculación cuyos rendimientos fueron estadísticamente inferiores, tuvieron un consumo más bajo de estos nutrientes. 
En los sistemas hidropónicos un porcentaje de los nutrientes son retenidos en el sustrato (Pineda et al., 2011) y pueden disociarse o precipitarse en función del $\mathrm{pH}$ o por efecto de su concentración en la solución nutritiva (DeRijck y Schrevens, 1998). Al usar arena de tezontle como sustrato, Pineda et al. (2011) reportaron que del total de nutrientes aplicados a un cultivo de tomate crecido durante $74 \mathrm{~d}$ en condiciones de hidroponía con drenaje sin recirculación de la solución nutritiva, $35.1 \%$ de K, 1.9 \% de N y $54.8 \%$ de $\mathrm{P}$ fueron retenidos por el sustrato.

Estos mismos autores agregaron que durante los primeros $40 \mathrm{~d}$ el sustrato almacenó gran cantidad de nutrientes, $y$ que fue hasta la fase de floración y el inicio de cuajado de fruto cuando parte de esos nutrientes retenidos en el sustrato comenzaron a ser liberados y absorbidos por la planta. En la presente investigación se usó el mismo sustrato que utilizó Pineda et al. (2011); al despuntar las plantas de pepino a $1 \mathrm{~m}$ de altura ya no hubo más crecimiento, por lo que una buena parte de los nutrientes quedaron retenidos en el sustrato (Cuadro 4) y así originaron un incremento de la CE en la solución drenada.

\section{CONCLUSIONES}

Entre los sistemas con y sin recirculación de la solución nutritiva que utilizaron sustrato y el mismo tipo de contenedor (bolsas o camas), el crecimiento y rendimiento de pepino en un ciclo corto donde las plantas se despuntaron a $1 \mathrm{~m}$ de altura, fueron similares. Sobresalió el sistema de raíz flotante por haber presentado mayor rendimiento por unidad de superficie en comparación con los sistemas de cultivo en bolsa. Lo anterior significa que con un manejo del cultivo de pepino en ciclos cortos mediante despunte temprano y plantado en altas densidades de población, es posible usar sistemas con recirculación de la solución nutritiva sin menoscabo del rendimiento, con respecto a sistemas sin recirculación.

En los sistemas con recirculación de la solución nutritiva se obtuvo un ahorro superior a $20 \%$ de agua y superior a 30 $\%$ en nutrimentos ( $\mathrm{N}, \mathrm{P}$ y K), comparado con sus sistemas similares sin recirculación.

\section{BIBLIOGRAFÍA}

Adams P. (2004) Aspectos de la nutrición mineral en cultivos sin suelo en relación al suelo. In: Tratado de Cultivo sin Suelo. G. M. Urrestarazu (ed). Ed. Mundi-Prensa. Madrid, España. pp:81111.

Alarcón V. A. (2006) Proyectos en cultivo sin suelo ¿Cómo empezar? In: Cultivos sin Suelo. V. A. Alarcón (ed.). Compendios de Horticultura 17. Ediciones de Horticultura, S. L. Reus. España. pp:11-21.

AMHPAC, Asociación Mexicana de Horticultura Protegida A. C. (2013) Mexico boasts nearly 21 thousand hectares under protected agriculture. http://www.houseofproduce.com/news/ production/?storyid=141 (Octubre 2013).
Cánovas M. F. y C. J. Magán (2003) Cultivos sin suelo. In: Técnicas de Producción en Cultivos Protegidos. F. F. Camacho (ed). Instituto Cajamar. Madrid, España. pp:409-453.

Castellanos J. Z. y M. C. Borbón (2009) Panorama de la horticultura protegida en México. In: Manual de Producción de Tomate en Invernadero. J. Z. Castellanos (ed). Ed. Intagri. Celaya, Guanajuato, México. pp:1-18.

Chapman S. R. and L. C. Pratt (1973) Crop Production. Principles and Practices. Ed. W.H. Freedman and Company. USA. 566 p.

De-Rijck G. and E. Schrevens (1998) Elemental bioavailability in nutrient solutions in relation to precipitation reactions. Journal of Plant Nutrition 21:203-2113.

Giuffrida F. and C. Leonardi (2009) Nutrient solution concentrations in soilless closed system. Acta Horticulturae 807:463-468.

Giuffrida F., V. Lipari and C. Leonardi (2003) A simplified management of closed soilless cultivation systems. Acta Horticulturae 614:155-160.

Huang W. Y. (2009) Factors Contributing to the Recent Increase in U.S. Fertilizer Prices, 2002-08. Agricultural Resources Situation and Outlook Number AR-33. U.S. Department of Agriculture, Economic Research Service, Washington, DC. 21 p.

Jones J. B. (2005) The plant root: its roles and functions. In: Hydroponics: A Practical Guide for the Soilless Grower. J. B. Jones (ed.). Ed. CRS Press. USA. pp:19-28.

Liang W., Y. Jiang and Y. Zhang (2006) Accumulation of soil soluble salt in vegetable greenhouses under heavy application of fertilizers. Agricultural Journal 1:123-127.

Massa D., L. Incrocci, R. Maggini, G. Carmassi, C. A. Campiotti and A. Pardossi (2010) Strategies to decrease water drainage and nitrate emission from soilless culture of greenhouse tomato. Agriculture Water Management 97:971-980.

Nakano Y., H. Sasaki, A. Nakano, K. Suzuki and M. Takaichi (2010) Growth and yield of tomato plants as influenced by nutrient application rates with quantitative control in closed rockwool cultivation. Journal of the Japanese Society for Horticultural Science 79:47-55.

Ortiz C. J., F. Sánchez-del-Castillo, C. Mendoza-Castillo y G. A. Torres (2009) Características deseables de plantas de pepino crecidas en invernadero e hidroponía en altas densidades de población. Revista Fitotecnia Mexicana 32:289-294.

Pardossi A., L. Incrocci, D. Massa, G. Carmassi and R. Maggini (2009) The influence of fertigation strategies on water and nutrient efficiency of tomato grown in closed soilless culture with saline water. Acta Horticulturae 807:445-450.

Parra M., V. Raya, M. C. Cid and J. Haroun (2009) Alternative to tomato soilless culture in open system in the Canary Islands: preliminary results. Acta Horticulturae 807:509-514.

Pineda P. J., A. A. Ramírez, F. Sánchez-del-Castillo, G. A. M. Castillo, A. L. A. Valdez and C. J. M. Vargas (2011) Extraction and nutrient efficiency during the vegetative growth of tomato under hydroponics conditions. Acta Horticulturae 893:997-1005.

Ponce C. P. (2013) Panorama de la Agricultura Protegida en México. http://www.hortalizas.com/articulo/35512/panorama-de-laagricultura-protegida-en-mexico (Julio 2013).

Raviv M. and H. Lieth (2008) Significance of soilless culture in agriculture. In: Soilless Culture Theory and Practice. M. Raviv and H. Lieth (eds.). Ed. Elsevier. Amsterdam, The Netherlands. pp:111.

Sánchez-del-Castillo F. y R. E. Escalante (1988) Hidroponía. Estudio de un Sistema de Producción. Ed. Universidad Autónoma Chapingo. Chapingo, México. $194 \mathrm{p}$.

Sánchez-del-Castillo F., E. del C Moreno-P., E. Contreras-M. y E. V. González (2006) Reducción del ciclo de crecimiento en pepino europeo mediante trasplante tardío. Revista Fitotecnia Mexicana 29:87-90.

Savvas D., N. Sigrimis, E. Chatzieustratiou and C. Paschalidis (2009) Impact of a progressive $\mathrm{Na}$ and $\mathrm{Cl}$ accumulation in the root zone on pepper grown in a closed-cycle hydroponic system. Acta Horticulturae 807:451-456.

Silber A. and A. Bar-tal (2008) Nutrition of substrate-grown plants. In: Soilless Culture Theory and Practice. M. Raviv and H. Lieth (eds.). Ed. Elsevier. Amsterdam, The Netherlands. pp:291-339.

Sonneveld C. and W. Voogt (2009) Substrates: chemical characteristics and preparation. In: Plant Nutrition of Greenhouse Crops. C. 
Sonneveled and W. Voogt (eds.). Springer. pp:227-252.

Terabayashi S., I. Muramatsu, S. Tokutani, M. Ando, E. Kitagawa, T. Shigemori, S. Date and Y. Fujime (2004) Relationship between the weekly nutrient uptake rate during fruiting stages and fruit weight of Tomato (Lycoperusicon esculentum Mill.) grown hydroponically. Journal of the Japanese Society for Horticultultural Science 73:324-329.
Tüzel I. H., U. Tunali, Y. Tüzel and G. B. Öztekin (2009) Effects of salinity on tomato in a closed system. Acta Horticulturae 807:457 462.

Van-Os E. A. (2009) Comparison of some chemical and non-chemical treatments to disinfect a recirculating nutrient solution. Acto Horticulturae 843:229-234. 